\title{
Temperature measurements on fast- rotating objects using a thermographic camera with an optomechanical image derotator
}

\section{Bettina Altmann, Christian Pape, Eduard Reithmeier}

Bettina Altmann, Christian Pape, Eduard Reithmeier, "Temperature measurements on fast-rotating objects using a thermographic camera with an optomechanical image derotator," Proc. SPIE 10404, Infrared Sensors, Devices, and Applications VII, 104040P (30 August 2017); doi: 10.1117/12.2271817

SPIE Event: SPIE Optical Engineering + Applications, 2017, San Diego, California, United States 


\title{
Temperature measurements on fast-rotating objects using a thermographic camera with an optomechanical image derotator
}

\author{
Bettina Altmann ${ }^{\mathrm{a}}$, Christian Pape $^{\mathrm{a}}$, and Eduard Reithmeier ${ }^{\mathrm{a}}$ \\ anstitute of Measurement and Automatic Control, Leibniz Universität Hannover, Nienburger \\ Str. 17, 30167 Hannover, Germany
}

\begin{abstract}
Increasing requirements concerning the quality and lifetime of machine components in industrial and automotive applications require comprehensive investigations of the components in conditions close to the application. Irregularities in heating of mechanical parts reveal regions with increased loading of pressure, draft or friction. In the long run this leads to damage and total failure of the machine. Thermographic measurements of rotating objects, e.g., rolling bearings, brakes, and clutches provide an approach to investigate those defects. However, it is challenging to measure fast-rotating objects accurately. Currently one contact-free approach is performing stroboscopic measurements using an infrared sensor. The data acquisition is triggered so that the image is taken once per revolution. This leads to a huge loss of information on the majority of the movement and to motion blur. The objective of this research is showing the potential of using an optomechanical image derotator together with a thermographic camera. The derotator follows the rotation of the measurement object so that quasi-stationary thermal images during motion can be acquired by the infrared sensor. Unlike conventional derotators which use a glass prism to achieve this effect, the derotator within this work is equipped with a sophisticated reflector assembly. These reflectors are made of aluminum to transfer infrared radiation emitted by the rotating object. Because of the resulting stationary thermal image, the operation can be monitored continuously even for fast-rotating objects. The field of view can also be set to a small off-axis region of interest which then can be investigated with higher resolution or frame rate. To depict the potential of this approach, thermographic measurements on a rolling bearings in different operating states are presented.
\end{abstract}

Keywords: Rotating machinery, Temperature measurements, Infrared imaging, Optomechanical image derotator, Rolling bearings

\section{INTRODUCTION}

Rotating machinery is widely spread in various fields of engineering and of major importance in industrial applications. To enable high efficiency of those machines, they are required to operate without interruption. For this, all rotating components have to function properly. In using different measurement techniques, parameters of condition of the machine (in- and out-of-plane movements, temperature, etc.) can be monitored. This affects varying machine elements for instance bearings, compressors, fans, or shafts.

This work focuses on temperature measurements of rolling bearings. Since a rise in temperature is linked to several rolling bearing damages, thermal imaging can be an effective method for fault diagnosis. Several authors investigated the connection between cage and roller slip and the temperature of the bearing. Slip is described by Boness [1] and Tassone [2] as a relative difference in the velocity between the rolling elements or the cage of the bearing: the predicted velocity of the elements differs from the actual velocity. This can happen due to a poor frictional force between the rolling-elements and the raceway. It can induce insufficient lubrication of the races. As a result, this can lead to wear of the races which manifests in spotted smear marks or roughness called skidding.

The influence of the outer race temperature and the oil viscosity on cage slip was examined by Smith [3].

Further author information:

E-mail: bettina.altmann@imr.uni-hannover.de, Telephone: +495117624280

Infrared Sensors, Devices, and Applications VII, edited by Paul D. LeVan, Ashok K. Sood,

Priyalal Wijewarnasuriya, Arvind I. D'Souza, Proc. of SPIE Vol. 10404, 104040P

(C) 2017 SPIE · CCC code: 0277-786X/17/\$18 - doi: 10.1117/12.2271817

Proc. of SPIE Vol. 10404 104040P-1 
Kacmarsky also reported on the effect of a different lubricant, a high transaction fluid, and the outer race temperature on cage slip [4]. Furthermore, Poplawski stated that an increase in oil temperature reduces cage slip [5]. The same was also concluded by Tassone who added that low oil flow can decrease slip but can increase skidding damage [2]. Selvaraj discussed the effect of different operating parameters on cage slip of cylindrical rolling bearings and, in this course, claimed that the correlation between increasing temperature and decreasing cage slip is due to the reduced viscosity [6]. An examination of the connection between temperature rise and skidding has been performed by $\mathrm{Li}$ [7]. He figured out that a rapidly increasing temperature can lead to skidding whereas a slowly increasing temperature does not evoke skidding.

There are different approaches on measuring the temperature of the respective bearing element. Earlier studies often used thermocouples or other contacting temperature sensors [3-6]. In recent studies infrared cameras (IR cameras) have been used to measure the temperature, e.g., in 7. Condition monitoring of the bearing's temperature using an IR camera has been conducted by various researchers, e.g., in 8 . The combination of an IR camera and a machine fault simulator has been pursued by $9,10,11$, and 12 .

In the course of this work, an IR camera will be used in combination with an optomechanical image derotator. This way, the temperature of the elements of the bearing can be measured in particularly and independently. An advantage is that the bearing can be measured in the operating state and in a non-contact manner. Furthermore, the bearing can be used in its original assembly and no changes have to be made on the components. Hence, different operating parameters and the influence on the temperature of the bearing can be investigated.

\section{OPTOMECHANICAL IMAGE DEROTATOR}

The predominant novelty in these investigations is the idea of combining a thermographic camera with the optomechanical image derotator. This enables thermographic measurements of a rotating object during operation. Moreover, the derotator compensates for the rotational velocity of the measurement object. Thus, the rotating object seems to stand still in the thermal images. As a result, the moving elements (e.g., the rolling elements, the cage or the inner/outer ring of a bearing) can be investigated precisely. The application of the derotator has the same result as if the thermographic camera would rotate synchronously with the measurement object. Derotator

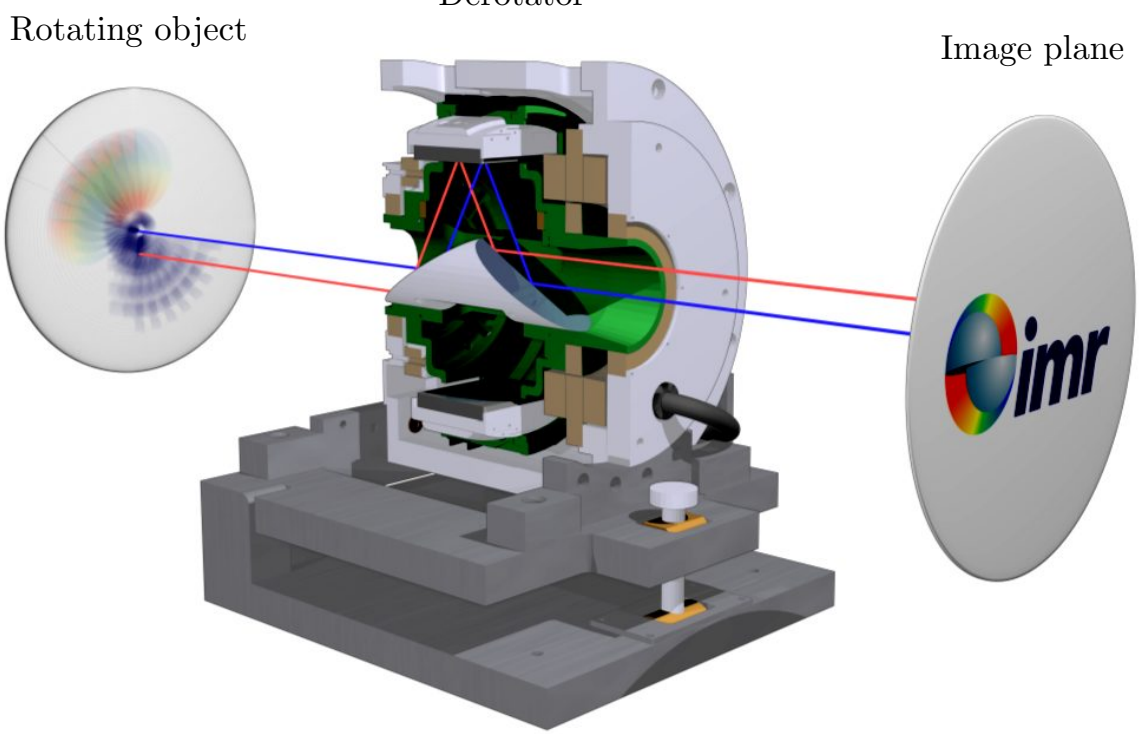

Figure 1: Assembly of the derotator with the reflector systems, the measurement object and the image plane.

However, the derotator uses optical elements to manipulate the beam path of the infrared radiation emitted by the object. The key component is a reflector system shown in Fig. 1. It consist of two individual parts: the first reflector is formed like a triangular prism where the two base areas are reflective. It is referred to as reflector prism. The second reflector is constructed as a common mirror and placed above the reflector prism. The assembly is inspired by reflective glass prisms like the Dove prism or the Abbe-Koenig prism, which are used 
to invert or rotate an image by refraction and reflection as shown in 13. Similarly, electromagnetic radiation is reflected by the reflector assembly three times in total. This is shown in Fig. 2. Since the reflectors are made of polished aluminum instead of glass, visible light as well as infrared radiation is reflected off the polished surfaces. In order to make use of the principle of the reflector assembly, it is places inside a hollow shaft motor (see also Fig. 1). Thus, the reflector assembly can be rotated around its own optical axis.

Nevertheless, two conditions have to be met to observe the effect of eliminating the rotational velocity of a measurement object. First, the reflector assembly has to rotate half as fast as the measurement object. Second, the optical axis of the reflector assembly and the rotational axis of the measurement object have to be aligned. For both problems, solutions have been developed throughout the recent years (see 14 and 15).

Accordingly, the adherence of the rotational velocity at a ratio of 1:2 is implemented using position control for the derotator. Thus, the angular position as well as the rotational velocity of the derotator are half as large as the corresponding parameters of the measurement object. The effect is pictured in Fig. 3: assuming the measurement object is rotated around an angle of $180^{\circ}$, the derotator and consequently the reflector assembly have to be rotated around $90^{\circ}$ to obtain a standing image in the image plane.

Furthermore, the alignment of the axes is implemented by using a parallel manipulator. Thus, the position of the derotator can be adjusted in six degrees of freedom. The automated calibration of the axes is performed by means of a mathematical model. It describes the residual movement of a point in the image plane. In identifying the parameters, a description of the miscalibration of the derotator is enabled. If the axes are identical, the movement of a point in the image plane is eliminated. Hence, the model is utilized in an optimization algorithm to align the optical axis of the derotator with the rotational axis of the measurement object.

Subsequently, the derotator can be combined with a measurement system to acquire measurement data of a rotating object. By respecting the aforementioned conditions, the images are free from the influence of the rotational velocity. Thus, a thermographic camera can produce very sharp thermal images of a rotating object and all its components.

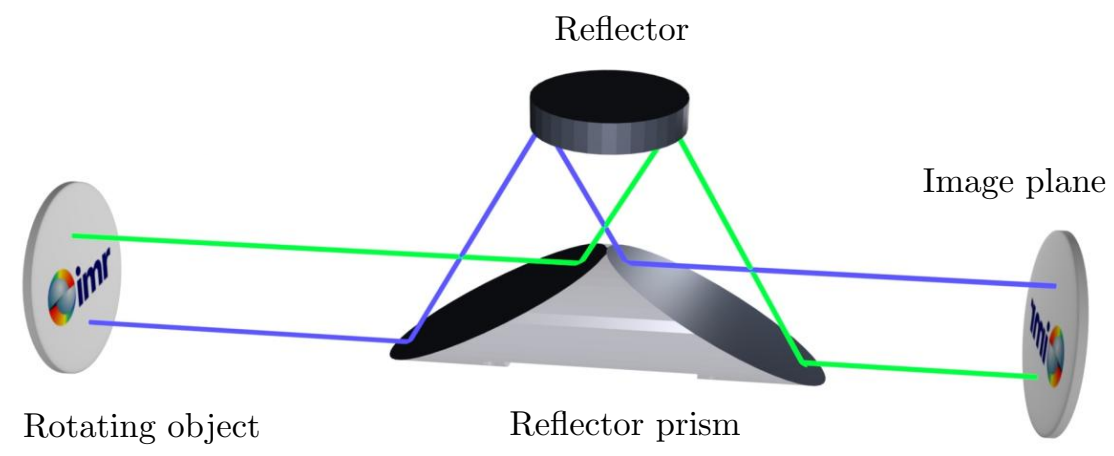

Figure 2: Optical path and assembly of the reflector system.

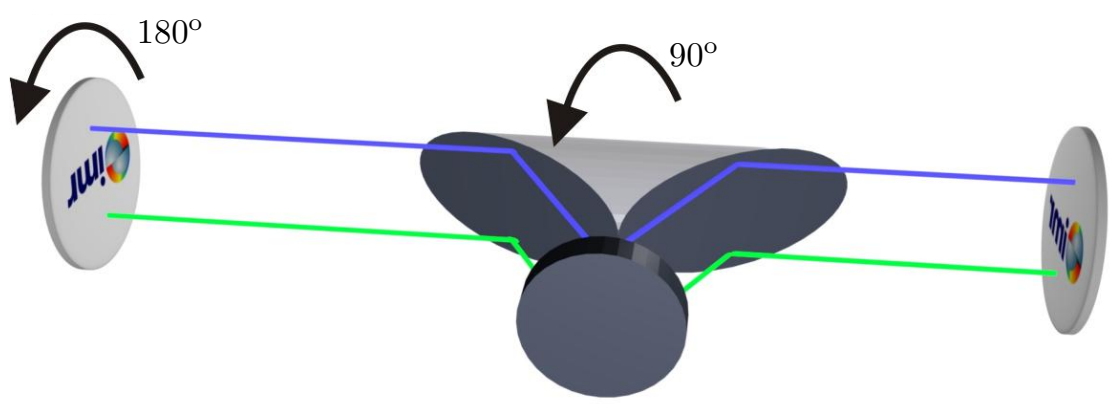

Figure 3: Effect of a rotation on the optical path and image. 
Table 1: Technical specifications of the three-phase-synchronous motor.

\begin{tabular}{|l|l|}
\hline Manufacturer & KOLLMORGEN \\
\hline Model & AKM44J-ANCNR-00 \\
\hline Max. speed & $6000 \mathrm{rpm}$ \\
\hline Peak torque & $20.4 \mathrm{Nm}$ \\
\hline Peak current & $35.2 \mathrm{~A}$ \\
\hline Torque constant & $0.69 \mathrm{Nm} / \mathrm{A}$ \\
\hline
\end{tabular}

\section{EXPERIMENTS}

Fig. 4 shows the experimental setup used in the investigations. It consist of the derotator (see Sec. 2), a bearing setup and a thermographic camera.

The test bearing is a SKF NU 208 ECP bearing with a cylindrical bore of 40mm, outer diameter of $80 \mathrm{~mm}$, and width of $18 \mathrm{~mm}$. There are 14 cylindrical rolling elements mounted on a cage which can carry a basic dynamic load of $62 \mathrm{kN}$. It is shown in Fig. 5. The inner ring of the bearing is actuated with a permanently excited three-phase-synchronous servo motor AKM44J powered with a servo drive AKD-P01207 both from Kollmorgen. The technical data is summarized in Tab. 1. On the other hand, the outer race is placed inside a mounting for fixation.

Furthermore, markers are applied on the bearing to determine the speed of the inner race, the cage and the rolling elements using image processing algorithms (see Fig. 5). In this way, dynamic effects of the bearing, e.g., roller or cage slip, can be identified. However, this is not further discussed in this paper but part of research in 16.
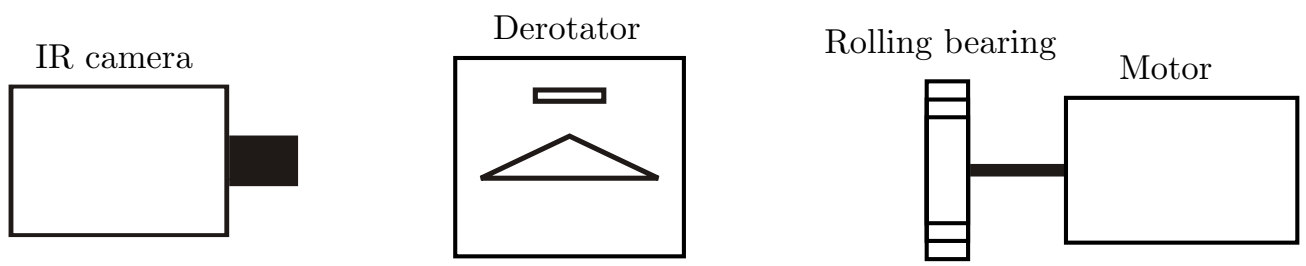

Figure 4: Schematic view of the experimental setup.

\subsection{Experimental setup}

To measure the infrared radiation emitted by the bearing during operation, a thermographic camera IMAGEIR 8380 from InFRATEC is used. Additionally, the IR camera can be complemented with severe interchangeable radiometric infrared lenses. Depending on the size of the frame, the camera can save images up to a frame rate of $850 \mathrm{~Hz}$. In these investigations, a precision radiometric infrared lens with a focal length of $25 \mathrm{~mm}$ is applied. Half frame images are acquired with a frame rate of $325 \mathrm{~Hz}$. Further technical data of the IR camera is displayed in Tab. 2.

\subsection{Experimental procedure}

In the following the measurement procedure will be depicted. Thus, the bearing is operated at two different rotational speeds: first, the bearing is driven with a rotational speed of $100 \mathrm{rpm}$. Second, the bearing is operated at a speed of $500 \mathrm{rpm}$. As a result, the cage of the bearing will travel with a speed of $40.5 \mathrm{rpm}$ or respectively $202.5 \mathrm{rpm}$.

Both speed profiles will be investigated with the IR camera and the derotator. To generate an image where the cage is standing still and the rolling elements rotate around the same center in the image plane, the derotator is running at a speed of $20.25 \mathrm{rpm}$ or respectively $101.25 \mathrm{rpm}$. 
Table 2: Technical specifications of the IR camera.

\begin{tabular}{|l|l|}
\hline Manufacturer & INFRATEC \\
\hline Model & ImageIR $\AA 8380$ \\
\hline Spectral range & $(2.0 \ldots .5,7) \mathrm{m}$ \\
\hline Temperature measuring range & $(-40 \ldots 1500)^{\circ} \mathrm{C}$ \\
\hline Measurement accuracy & $\pm 1^{\circ} \mathrm{C}$ or $\pm 1 \%$ \\
\hline Frame rate (full/half/quarter frame) & $\mathrm{Up}$ to $100 / 355 / 850 \mathrm{~Hz}$ \\
\hline
\end{tabular}

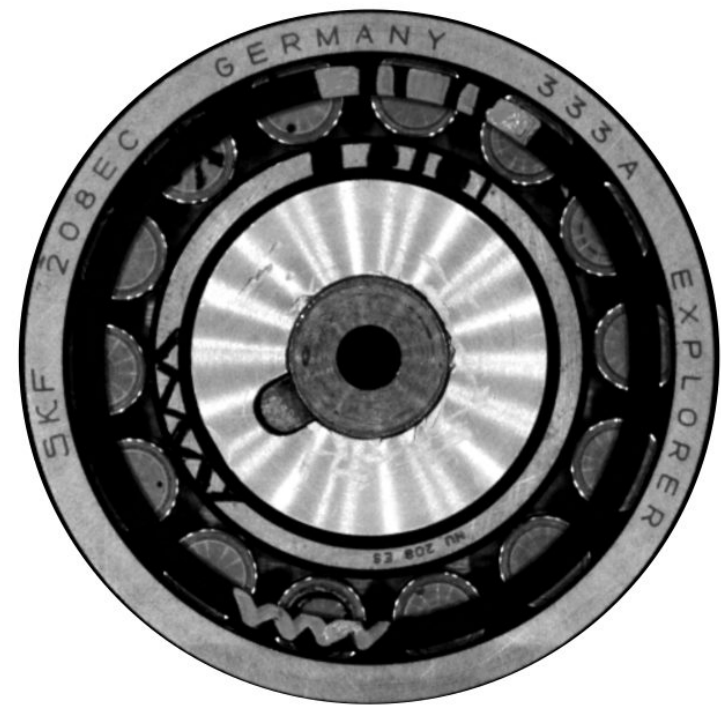

Figure 5: Rolling bearing used in the experiments.

Each experiment is performed for $300 \mathrm{~s}$ in total. It is expected, that the temperature of the bearing elements increases with the speed. Furthermore, it should be investigated if the derotator enables sharp and precise thermal images of the bearing and its elements. Consequently, the measurements will be discussed and compared to each other.

To produce exactly defined thermal images, the thermal conductivity of each material of the bearing has to be known. However, this is challenging and therefore has not yet been done up to the time of the temperature measurements in this paper. Subsequently, the thermal images are assessed in qualitative terms. Due to its functional principle the derotator enables investigations of the stationary thermal distribution of the complete bearing.

\section{APPLICATION AND RESULTS}

In the following, the results of the experiments specified in Sec. 3 will be clarified. Fig. 6 and 7 picture measurements on a bearing at three different points in time: Fig. 6a and 7a show the heat map at the beginning of the measurement, Fig. $6 \mathrm{~b}$ and $7 \mathrm{~b}$ in the middle at $t=150 \mathrm{~s}$, and Fig. $6 \mathrm{c}$ and $7 \mathrm{c}$ in the end at $t=300 \mathrm{~s}$.

In each picture the bearing elements are clearly visible. This is possible due to the elimination of the rotational velocity with the help of the derotator which prevents motion blur. The rolling elements are clearly distinguishable from the background of the bearing as well as the cage is clearly distinguishable from the rolling elements. Furthermore, even some markers placed on the rolling elements are visible. This is due to the different thermal conductivity of the marker. Thus, the speed of the rolling elements could be determined from the thermal images. 


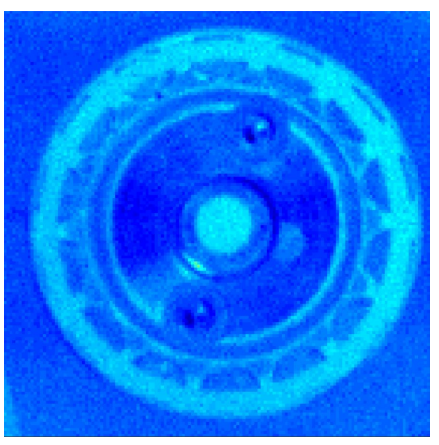

(a) At $t=0 \mathrm{~s}$.

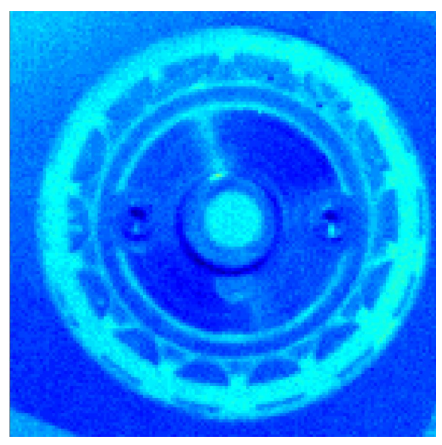

(b) At $t=150 \mathrm{~s}$.

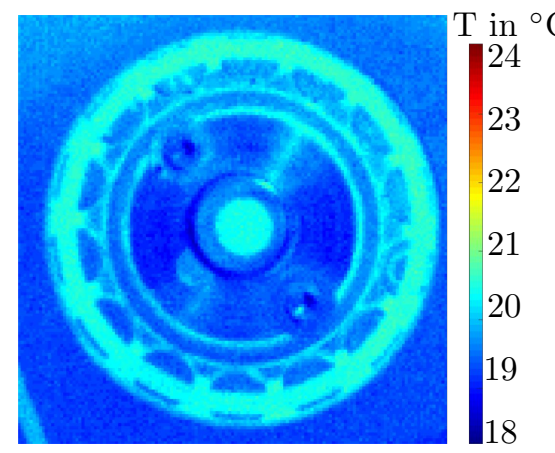

(c) At $t=300 \mathrm{~s}$.

Figure 6: Temperature measurements at a speed of $100 \mathrm{rpm}$.

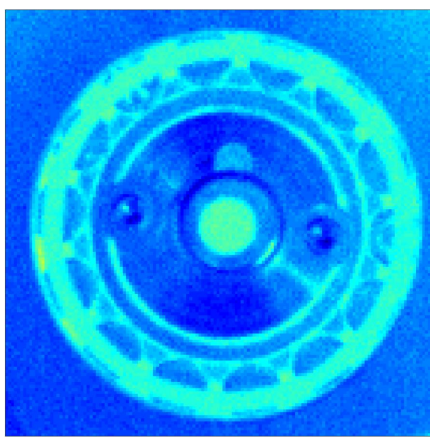

(a) At $t=0 \mathrm{~s}$.

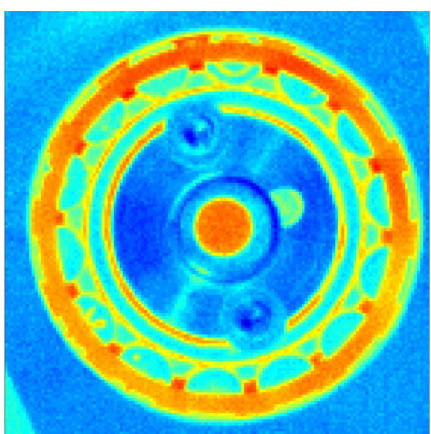

(b) At $t=150 \mathrm{~s}$.

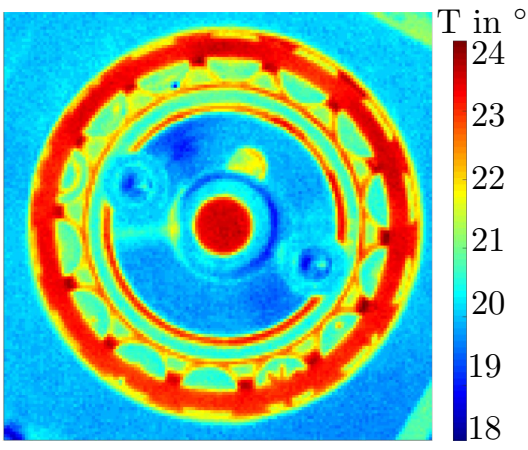

(c) At $t=300 \mathrm{~s}$.

Figure 7: Temperature measurements at a speed of $500 \mathrm{rpm}$.

When looking at the first measurement procedure at $100 \mathrm{rpm}$ (see Fig. 6) it can be observed that the cage is changing its temperature faster than the rolling elements. The temperature goes up from about $19.9{ }^{\circ} \mathrm{C}$ to $20.7^{\circ} \mathrm{C}$. Furthermore, the contact areas between the rolling elements and the inner/outer race and the inner race and the adapter for the shaft are warming up. This can be explained by the friction between the contact partners: kinetic energy is transformed to thermal energy. However, the temperature of the center of the rolling elements remains relatively constant.

In the second case, the rolling bearing is operated at $500 \mathrm{rpm}$ (see Fig. 7). As well as in the first case, the cage is changing its temperature faster except that it is going up to about $23.3{ }^{\circ} \mathrm{C}$. The same applies to the contact areas of all rotating elements. Since the rotational velocity is higher in this case, the kinetic energy and therefore the thermal energy and heat development are higher, too. Additionally, the rolling elements are heating up from round about $19.6{ }^{\circ} \mathrm{C}$ to $20.7^{\circ} \mathrm{C}$. Since the temperature gradient is higher in this case, the thermal conduction between the elements is higher, too. This explains, why also elements are heating up which not directly undergo friction.

\section{CONCLUSION}

In sum, it can be said that the derotator is an effective system to improve measurements on rotating system. Due to its operating principle, motion blur is prevented. This is particularly relevant when the bearing is operating at higher velocities. Thus, thermal images of fast rotating objects, e.g., bearings are enabled. For future investigations the thermal conductivity of each material can be determined, so that the measurements are both quantitative and qualitative.

In this paper, an example measurement comparing a bearing rotating at two different rotational velocities has been shown. The diverse bearing elements are easily distinguishable from each other, so that the temperature of each element can be determined separately. This is of considerable interest, when different operating states of the bearing are investigated: in case different lubricants are compared to each other it is directly visible which 
lubricant is more suitable for a certain application.

For future experiments more operating parameters can be varied: the influence of, e.g., clearance assembly, radial loads, higher rotational velocities, and lubricant viscosity can be examined. Thus, a connection between heat development and different scenarios can be determined.

Furthermore, measurements on cage and roller slip can be contrasted with thermal images which were acquired simultaneously. Hence, a direct correlation between slip and friction/heat transfer can be demonstrated. In case a particular rolling element has a defect, it could be monitored if there is a relation between both parameters. For this, the lens of the IR camera can be changed to one with higher magnification. Thus, the field of view can also be set to a small off-axis region of interest which then can be investigated with higher resolution.

\section{ACKNOWLEDGMENTS}

The authors would like to thank the BMWi for funding the project "GAeroFeRo". Special thanks go to our colleague Waldemar Gorschenew.

\section{REFERENCES}

[1] Boness, R. J., "Cage and roller slip in high-speed roller bearings," Journal of Mechanical Engineering Science 11(2), 181-188 (1969).

[2] Tassone, B. A., "Roller bearing slip and skidding damage," Journal of Aircraft 12(4), 281-287 (1975).

[3] Smith, C. F., "Some aspects of the performance of high-speed lightly loaded cylindrical roller bearings," Wear 5(3) (1962).

[4] Kacmarsky, W. M. and Hewko, L. O., "Effect of a High Traction Fluid on Skidding in a High Speed Roller Bearing," Journal of Lubrication Technology 93(1), 11-16 (1971).

[5] Poplawski, J. V., "Slip and Cage Forces in a High-Speed Roller Bearing," Journal of Lubrication Technology 94(2), 143-150 (1972).

[6] Selvaraj, A. and Marappan, R., "Experimental analysis of factors influencing the cage slip in cylindrical roller bearing," The International Journal of Advanced Manufacturing Technology 53(5-8), 635-644 (2011).

[7] Li, J., Chen, W., and Xie, Y., "Experimental study on skid damage of cylindrical roller bearing considering thermal effect," Proceedings of the Institution of Mechanical Engineers, Part J: Journal of Engineering Tribology 228(10), 1036-1046 (2014).

[8] Janssens, O., Schulz, R., Slavkovikj, V., Stockman, K., Loccufier, M., v. d. Walle, R., and v. Hoecke, S., "Thermal image based fault diagnosis for rotating machinery," Infrared Physics Technology 73 (2015).

[9] Lim, G. M., Ali, Y., and Yang, B. S., "The Fault Diagnosis and Monitoring of Rotating Machines by Thermography," in [Engineering Asset Management and Infrastructure Sustainability], 557-565 (2012).

[10] Widodo, A., Satrijo, D., Prahasto, T., Lim, G. M., and Choi, B. K., "Confirmation of Thermal Images and Vibration Signals for Intelligent Machine Fault Diagnostics," International Journal of Rotating Machinery (2), 1-10 (2012).

[11] Younus, A. M. and Yang, B. S., "Intelligent fault diagnosis of rotating machinery using infrared thermal image," Expert Systems with Applications 39(2), 2082-2091 (2012).

[12] Tran, T., Yang, B. S., Gu, F., and Ball, A., "Thermal image enhancement using bi-dimensional empirical mode decomposition in combination with relevance vector machine for rotating machinery fault diagnosis," Mechanical Systems and Signal Processing 38(2), 601-614 (2013).

[13] Swift, D. W., "Image rotation devices: A comparative survey," Optics \& Laser Technology 4, 175-188 (1972).

[14] Altmann, B., Rohloff, B., Pape, C., and Reithmeier, E., "Identification and Tracking-Control for an Optomechatronical Image Derotator Using Neural Networks," PAMM 16(1), 795-796 (2016).

[15] Rohloff, B., Pape, C., and Reithmeier, E., "Automated calibration of an optomechanical derotator using 6-axes parallel kinematics and industrial image processing algorithms," Optical Engineering 53(10) (2014).

[16] Altmann, B., Pape, C., and Reithmeier, E., "Digital image processing algorithms for automated inspection of dynamic effects in roller bearings," in [SPIE Optical Metrology], SPIE (2017). 